Rabaska

Revue d'ethnologie de l'Amérique française

RABASKA

\title{
Un Américain à Saint-Denis
}

An American in Saint-Denis

\section{René Gagnon}

Volume 16, 2018

URI : https://id.erudit.org/iderudit/1051326ar

DOI : https://doi.org/10.7202/1051326ar

Aller au sommaire du numéro

Éditeur(s)

Société québécoise d'ethnologie

ISSN

1703-7433 (imprimé)

1916-7350 (numérique)

Découvrir la revue

Citer cet article

Gagnon, R. (2018). Un Américain à Saint-Denis. Rabaska, 16, 77-97. https://doi.org/10.7202/1051326ar

\section{Résumé de l'article}

Quelles traces laisse le passage d'un anthropologue ou d'un ethnologue dans une communauté ? De quelle façon la population sous observation reste-t-elle marquée ? Et l'observateur, lui, garde-t-il des marques ? L'article suivant et son annexe proposent bien modestement quelques réponses à ces questions. Revenant sur le passage d'Horace Miner à Saint-Denis-De La Bouteillerie, un village rural du Bas-Saint-Laurent, en 1936-1937, le descendant d'une des familles sous observation remonte le fil du temps à partir de l'album photographique de ses grands-parents. Puis, en annexe, Agnès Miner, l'épouse de l'anthropologue, revient sur le séjour du couple dans une communauté paysanne du Canada français des années 1930. 


\title{
Un Américain à Saint-Denis
}

\author{
RENÉ GAGNON \\ Société d'histoire et de généalogie de la Côte-du-Sud
}

Avril 2017 : au bénéfice de ses membres, la Société québécoise d'ethnologie présente Le Temps et le lieu de Bernard Émond, un documentaire qui traite, entre autres, du passage de l'Américain Horace Miner à Saint-Denis en 19361937'. L'auteur de cet article, qui assiste à la représentation, fait partie de la famille Gagnon, présente autant dans la monographie que Miner tira de son séjour que dans le film de Bernard Émond. Il revient ici sur les traces laissées par l'anthropologue dans le petit village du Bas-Saint-Laurent.

J'avais cinq ans, je n'allais pas encore à l'école, et pourtant ce moment est resté profondément gravé dans ma mémoire. Par un beau dimanche d'été, quelques douzaines de personnes emplissaient le spacieux séjour de la maison de mon grand-père Arthur. Assises en rond, elles conversaient dans le calme, avec une réserve certaine, voire avec gravité. Dans mon innocence d'enfant, je sentais bien la solennité du moment sans toutefois comprendre ce qui lui conférait ce caractère. Des gens importants étaient venus de loin et mes parents, surtout mon père (Arthur fils), qui habitaient eux aussi le rang du Coteau, quatre maisons à l'ouest de mes grands-parents, avaient tenu à participer à l'événement en famille. Mes sept frères et sœurs s'y trouvaient donc.

Le vif souvenir que je garde de l'événement n'est pas lié aux visiteurs. En fait, avec le temps j'ai oublié leurs traits, leur voix, leur allure, leur habillement et tout le reste. Ma mémoire s'est plutôt accrochée au plaisir que j'éprouvai cet après-midi-là à vivre une expérience à haute teneur affective : ma tante Laure m'avait mis entre les mains une bande dessinée appartenant à sa fille Lise, ma cousine, et elle s'amusait à me faire parler, à me faire raconter l'histoire narrée dans la bd. Je ne savais pas lire, mais les images et les échanges avec ma tante remplissaient mon univers.

Quand sonna l'heure du départ, tout le monde sortit sur la véranda pour raccompagner nos visiteurs à leur voiture. Ils avaient un long chemin à faire

1. Le Temps et le lieu, Québec, Coop Vidéo de Montréal. Réalisateur et scénariste : Bernard Émond ; productrice : Lorraine Dufour, 1999, 50 min. 
puisqu'ils venaient des États-Unis, mais peut-être n'y retournaient-ils pas directement, allez savoir. En 1959, cette visite serait la dernière qu'Horace Miner rendrait à mon grand-père, celui qu'il appelait « mon oncle Arthur ». Son épouse Agnes l'accompagnait de même que leur fille Denise, alors étudiante à l'université Stanford. Même s'il devait le comprendre encore un peu, l'anthropologue américain ne parlait presque plus français, selon mon frère Jacques, tandis que sa femme se débrouillait encore assez bien. Mon père avait accordé une grande importance à notre présence chez mon grand-père parce qu'il gardait une très forte impression du séjour de Miner à Saint-Denis en 1936-1937. D'ailleurs, cette relation initiale devait laisser des traces profondes autant sur les Miner que sur les Gagnon. L'anthropologue allait se transformer en personnage quasi légendaire dans ma famille tandis que sa conjointe et lui-même resteraient marqués de façon durable par cette première expérience de terrain.

\section{Sur le terrain}

Pour mon père, fier cultivateur qui n'avait pas fréquenté l'école au-delà de sa septième année, les livres avaient une grande valeur. De même que pour ma mère, maîtresse d'école jusqu'à son mariage. À deux, ils avaient constitué une bibliothèque qui occupait tout un pan de mur. Quand un relâchement de ses tâches le permettait, mon père lisait. Des almanachs, des ouvrages historiques, des essais politiques, des biographies. Il était abonné à L'Action catholique, puis au Soleil, ainsi qu'à des périodiques comme MacLean's ou Sélection du Reader's Digest - dont il lui manquait à peine quelques numéros. Ma mère, qui ne pouvait se passer de lecture, préférait les romans. Du plus loin que je me souvienne, la bibliothèque familiale ne comptait qu'un seul livre anglais : St. Denis, a French-Canadian Parish ${ }^{2}$, édition de 1963, avec une dédicace manuscrite de l'auteur. J'ignore comment mon père se l'était procuré. Chose certaine, il ne parlait ni ne lisait l'anglais. Il connaissait sans doute un certain nombre de mots, mais ne comprenait pas assez pour pleinement appréhender ce que H. Miner expose dans sa monographie. Malgré tout, il considéra toujours ce livre comme un bien précieux et nous parla souvent de l'anthropologue et de son séjour dans la paroisse. Même si l'événement remontait à plusieurs décennies, il en avait conservé de nombreux souvenirs dont il nous faisait part à l'occasion.

Après que mon frère aîné Jacques eut passé quelque temps aux États-Unis, mon père et lui convinrent de la nécessité de traduire St. Denis pour savoir

2. Horace Miner, St. Denis, a French-Canadian Parish, Chicago, The University of Chicago Press, « Phoenix Books» 1 108, 1963, xix-299 p. Cette réédition, en tous points conforme à l'édition originale, a été enrichie d'une postface (chapitre xII, p. 255-269) : Horace Miner, St. Denis, a FrenchCanadian Parish, Chicago, The University of Chicago Press, « Ethnological Series », 1939, xix-283 p. 
ce qu'on disait du village et de ses habitants. Mais mon frère ne parvint pas à consacrer le temps et l'énergie nécessaires à cette tâche. Il en traduisit une vingtaine de pages tout au plus.

Adolescent, j'avais de la facilité en anglais, aussi m'arrivait-il de jeter un coup d'œil au livre et d'en déchiffrer des passages. Mais les pauvres moyens linguistiques et théoriques dont je disposais ne me permettaient pas de saisir l'ampleur de l'œuvre. Il reste qu'Horace Miner dut exercer une influence sur moi sans que j'en aie pleine conscience puisque je décidai de consacrer une partie de mon programme d'études à l'anthropologie lors de mon inscription à l'université. Alors que rien d'autre ne m'y disposait. Assez vite, dès la deuxième session du bac, la monographie de Miner se retrouva en référence dans l'un quelconque de mes cours. Je compris beaucoup mieux alors ce qu'il avait fait à Saint-Denis et j'en éprouvai une certaine fierté. D'autant qu'il s'agit encore aujourd'hui, avec plus de quatre-vingts ans de recul, d'un ouvrage remarquable, voire magistral.

Quelques années plus tard, le goût de traduire St. Denis, a FrenchCanadian Parish me vint, à moi aussi. J'écrivis donc à l'auteur pour motiver ma requête et pour lui demander son autorisation. À mon grand désarroi, il me répondit :

Les raisons qui militant en faveur d'une traduction dès à présent ont, je suis désolé de le dire, déjà incité le professeur Jean-Charles Falardeau de l'Université Laval à prendre les dispositions pour publier le livre en français. Une première version de la traduction devrait être livrée ce mois-ci, mais la production du livre prendra plus d'un an, selon ce qu'on m'en a dit. ${ }^{3}$

\section{La mémoire affective}

La version française de St. Denis allait paraître en $1985^{4}$. Le livre profita d'une efficace mise en marché et d'un accueil critique favorable. On en fit des recensions élogieuses et il trouva une plus vaste audience dans son terroir qu'il n'en avait eue jusque-là. Toutefois, les Marcel Rioux, Jean-Charles Falardeau, Fernand Dumont ou Marc-Adélard Tremblay n'avaient pas attendu la traduction pour y faire écho et pour l'introduire dans les facultés universitaires de sciences sociales. C'est ainsi que Bernard Émond, le réalisateur du film Le Temps et le lieu, prit connaissance du texte pendant ses études en

3. Horace Miner à René Gagnon, lettre écrite de sa résidence d'été, Fryeburg (Maine), 13 août 1979 : «The very factors you mention as making such a translation desirable at this time have, I am sorry to say, already led Professor Jean-Charles Falardeau of Laval University to arrange for the publication of the book in French. The first draft of the translation should be completed this month, but the actual production of the book will take over a year I am told. » [Traduction de l'auteur de l'article]

4. Horace Miner, Saint-Denis : un village québécois, Présentation de Jean-Charles Falardeau, traduit de l'anglais par Édouard Barsamian et Jean-Charles Falardeau, Ville Lasalle, Hurtubise HMH, « Sciences de l'homme et humanisme» 11, 1985, $392 \mathrm{p}$. 
anthropologie. Et que l'idée d'en faire un film s'imposa à lui petit à petit. En partie, l'œuvre d'Émond retrace le parcours d'Horace Miner à Saint-Denis grâce à des informateurs que le cinéaste a dénichés et qui, tout jeunes encore, avaient eu l'occasion de rencontrer l'Américain, de l'apercevoir à la messe, sur le perron de l'église ou ailleurs. Un des intérêts du film repose sur la confrontation de marques intangibles révélées par le déploiement d'un appareil mémoriel - tant chez Agnes Miner que chez les informateurs locaux -, avec des éléments tangibles, sous la forme du livre, des photos, des notes de terrain, des extraits de lettres d'Agnes à son amie Bessie.

La qualité de la monographie de Miner tient à la justesse exceptionnelle de sa description de la société rurale traditionnelle du Québec. L'auteur y démontre une grande sagacité dans la sélection des exemples qui lui paraissent les plus pertinents parmi les individus et les groupes qu'il fréquente. Les Gagnon, au même titre que d'autres familles retenues, furent observés et analysés pour comprendre et mettre en lumière la nature des mécanismes psychologiques et sociaux qui rendent l'individu fonctionnel dans des ensembles plus vastes, tels la famille, le rang, la paroisse, la communauté des agriculteurs, aussi bien que dans des phases de la vie ou dans d'autres comportements humains, religieux ou politiques. Au sujet de ma famille en particulier, Horace Miner m'écrivait :

Vous reconnaissez probablement l'«Autobiographie d'un habitant» (Appendice III) comme celle de votre grand-père Arthur. L'analyse de la famille, p. 201 à 205 , porte aussi sur votre famille. Toutes les références à votre père sont très flatteuses, mais si vous y voyez quoi que ce soit de désobligeant, signalez-le-moi s'il vous plaît. ${ }^{5}$

Une question se pose alors : comment a-t-il réussi à marquer les gens aussi profondément tout en s'immisçant dans leur vie, en scrutant à la loupe le moindre de leurs faits et gestes?

Considérons d'abord l'impact affectif de son approche. L'attitude ouverte d'Horace Miner, ses rapports chaleureux avec la population de Saint-Denis, son insatiable curiosité tant pour les gens, leur point de vue, leurs activités, leurs croyances, leur histoire, leurs coutumes, etc., lui gagnèrent la sympathie des villageois. On peut présumer que sa volonté sincère de tout connaître sans jamais juger ni critiquer ni remettre en question finissait par lui gagner la bienveillance même des plus récalcitrants. "Toutte l'intéressait » insiste l'un des protagonistes du film Le Temps et le lieu. Si cet intérêt inquisiteur

5. Horace Miner à René Gagnon, lettre du 13 août 1979 : "You probably recognize "The Autobiography of an Habitant " (Appendix III) as that of your grandfather, Arthur. The family analysis on pp. 201-05 also involves your family. All references to your father are very complimentary, but if there is anything in that material which you feel is objectionable, please let me know. » [Traduction de l'auteur de l'article] 
s'avère opportun pour l'anthropologue, il comporte un appréciable pouvoir de gratification pour la population sous observation.

Mais il y a plus : peu de temps après son arrivée sur le terrain, Miner entreprit d'étudier les structures de parenté locales. Cette démarche fondamentale en anthropologie se révéla d'autant plus appropriée à Saint-Denis que l'habitant typique, comme mon père, mon grand-père ou leurs voisins, entraient en contact avec tout nouvel interlocuteur en détricotant sur deux, trois ou quatre générations les rapports de parenté plus ou moins lointains que leurs ancêtres avaient noués. Aujourd'hui encore, replongé dans le lieu de mes origines, je me surprends parfois à aborder les gens de cette manière. Quand je refais connaissance avec des personnes du coin, il est souvent question de leur filiation et de notre potentielle parenté, selon leur patronyme. Sommesnous cousins du deuxième ou du troisième degré, par quel ancêtre maternel ou paternel sommes-nous liés, etc. ? Miner élabora l'arbre généalogique de plusieurs familles, remontant parfois jusqu'en sol français. Entre autres, ses notes contiennent un schéma du patrilignage de mon grand-père à partir de ses racines percheronnes ${ }^{6}$.

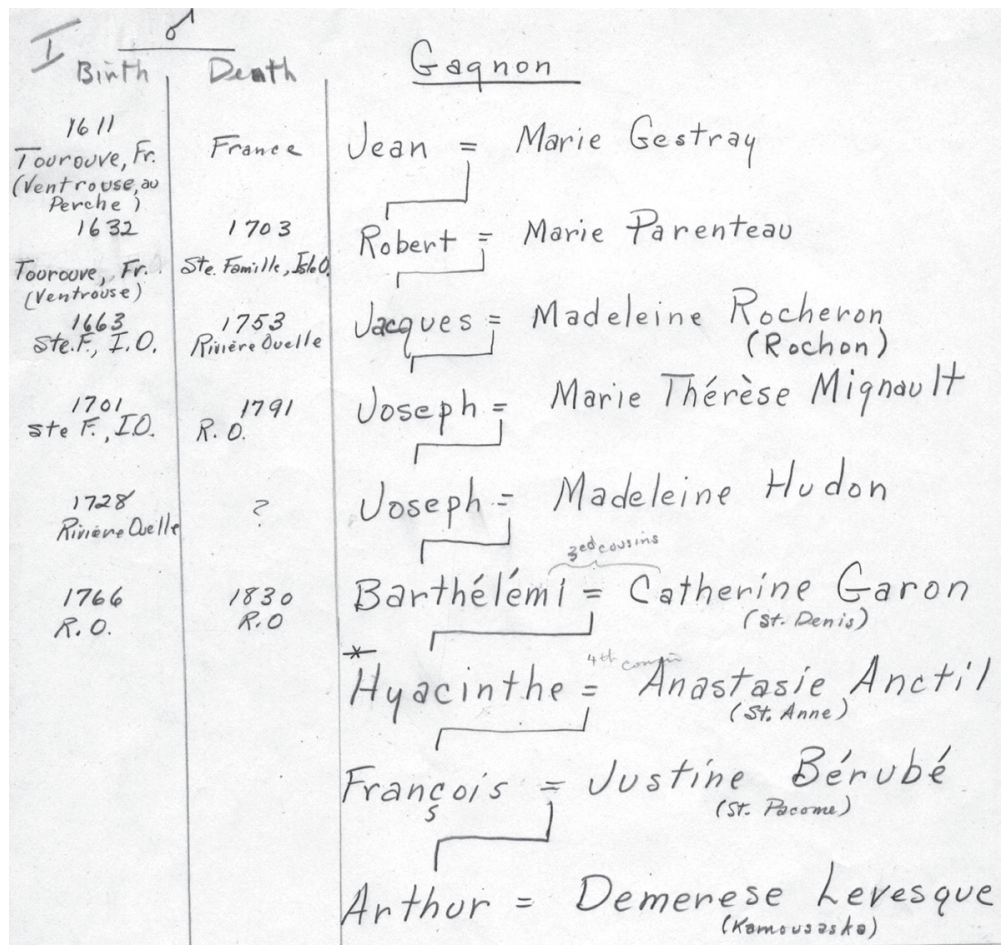

Patrilignage de mon grand-père, Arthur Gagnon

Document conservé aux Archives de la Côte-du-Sud

6. Archives de la Côte-du-Sud (ACS), Fonds 241 - Archives Horace Miner. 
Au départ, Horace Miner dut effectuer quelques recherches généalogiques, puis, ayant perçu l'intérêt des gens de Saint-Denis, il s'appliqua à les aborder sur le sujet de la parenté. Il améliorait ainsi la qualité de ses échanges dans une paroisse où, comme dans le cas de mon père et de mon grand-père, certains pouvaient remonter sur plusieurs générations, parfois jusqu'à leur origine française. L'anthropologue ne s'y trompe pas puisqu'il place l'attachement aux origines, à l'histoire et aux liens de parenté au cœur de l'identité canadienne-française : «Les Canadiens français sont fiers de leur histoire. Ils l'inculquent à leurs enfants et ils exhortent chaque nouvelle génération à suivre les traces de leurs ancêtres. ${ }^{7}$ $"$ Nul doute qu'avec une telle approche, Miner se fit de nombreux alliés, complices et amis dans le village. Il ouvrait des portes lui permettant de pousser plus loin ses relations avec les familles et chacun de leurs membres. À la limite, on finissait par l'adopter : « Comme votre grand-père Arthur m'avait permis de l'appeler mon oncle Arthur, vous et moi devons être un genre de cousins $!^{8}{ }^{\prime \prime}$

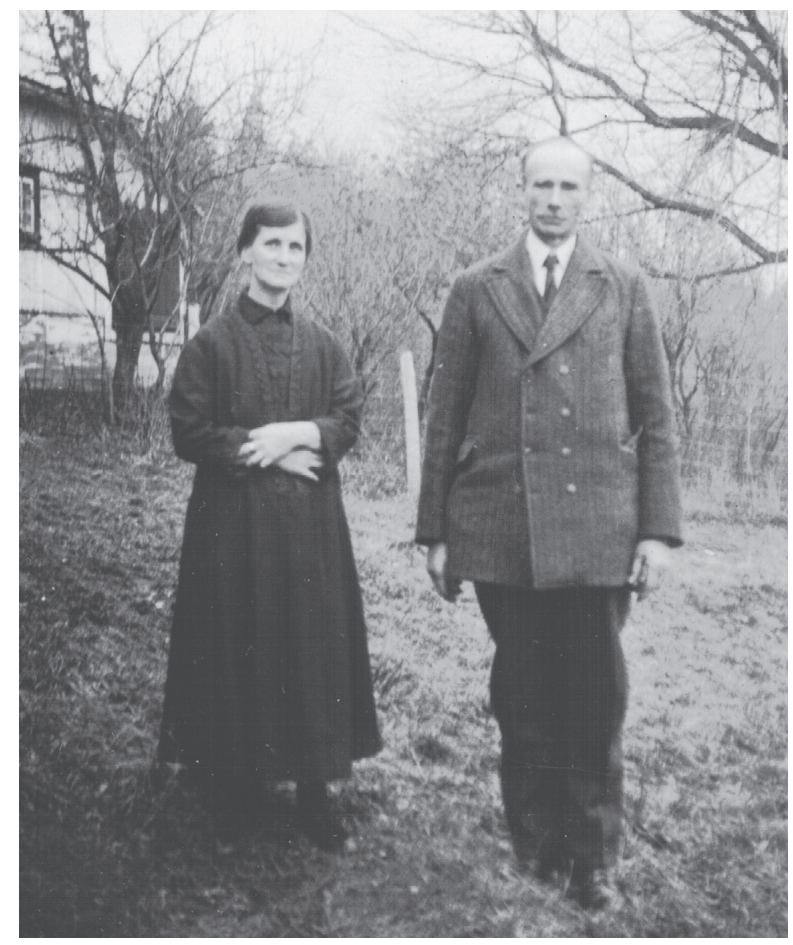

Ma grand-mère Demerise et mon grand-père Arthur

Horace Miner les appelait « ma tante » et «mon oncle Arthur »

7. Horace Miner, Saint-Denis : un village québécois, op. cit., p. 31.

8. Horace Miner à René Gagnon, lettre du 13 août 1979: «As your grand-père Arthur permitted me to call him mon oncle Arthur, you and I must be cousins of some sort! ». [Traduction de l'auteur de l'article] 


\section{Les images comme cailloux blancs}

L'anthropologue était réputé trimballer sa caméra et photographier tout ce qu'il jugeait digne d'intérêt. On le vit partout participer aux événements quotidiens, observer les travaux des cultivateurs et des artisans, assister aux cérémonies religieuses. Et tout le temps prendre des photos. Dans Le Temps et le lieu, un informateur se souvient : «Ça, sa caméra, ça le suivait tout le temps. » Comme s'il semait des cailloux blancs en chemin, il prenait des photos pour remonter, après coup, le fil de ses souvenirs. Pour un chercheur objectif et intègre, la photographie est un moyen de reproduire la réalité au plus près, sans biais. La factualité du visuel vient corriger la subjectivité et les défaillances de la mémoire. Les images lui servaient en quelque sorte d'adjuvant à ses notes de terrain puisqu'il ne notait rien sur-le-champ. Entre autres utilités, elles lui servaient à saisir la fugacité d'un moment ou, au contraire, à définir des repères grâce auxquels il pourrait plus tard, au stade de l'analyse ou de la rédaction, approfondir des idées, des raisonnements ou des hypothèses.

Il est arrivé à Horace Miner de composer des images avec l'idée originale de représenter une structure sociale. Par exemple, il décrit une structure hiérarchique domestique, celle d'une maisonnée - soit les membres d'une famille partageant le quotidien dans une maison -, avec le rôle et la place de chacun dans la dynamique globale ${ }^{9}$. Il existe une photographie, comme un écho de cette description, qui présente graphiquement les rapports d'autorité dans la maisonnée de mon grand-père.

Cette image n'était pas destinée à la publication ni au lecteur ni au directeur de recherche (Robert Redfield) du doctorant. Il la composa pour son usage personnel, comme aide-mémoire illustrant l'exercice de l'autorité dans la maison, tel qu'expliqué dans un chapitre sur l'enfance. On a donc affaire ici à une photo concept. Dans St. Denis, la description des personnages, de leur personnalité et de leur place dans la maison se suffit à elle-même, sans qu'il soit besoin de l'appuyer sur une béquille graphique. On peut même croire qu'il s'agit d'une famille complète. Or, la photo compte seulement huit des quatorze enfants de la famille Gagnon; il en manque six : les trois aînés, François, Marie-Louise et Jean-Charles, respectivement prêtre, religieuse et frère, ne vivaient plus dans la maison familiale depuis des années ; MarieThérèse, 23 ans à l'époque, travaillait à Québec comme infirmière tandis que les jumelles Anne et Jeanne, 16 ans, étaient pensionnaires dans une école ménagère. Il ne s'agit donc pas de hasard : Miner a fait d'autres photos de la famille Gagnon dont une où les quatorze enfants sont rassemblés dans le salon pour une photo de famille très « officielle ». La conceptualisation de relations d'autorité ainsi mise en image manifeste le remarquable sens

9. Horace Miner, St. Denis, op. cit., p. 201-205 ; Saint-Denis, op. cit., p. 262-268. 


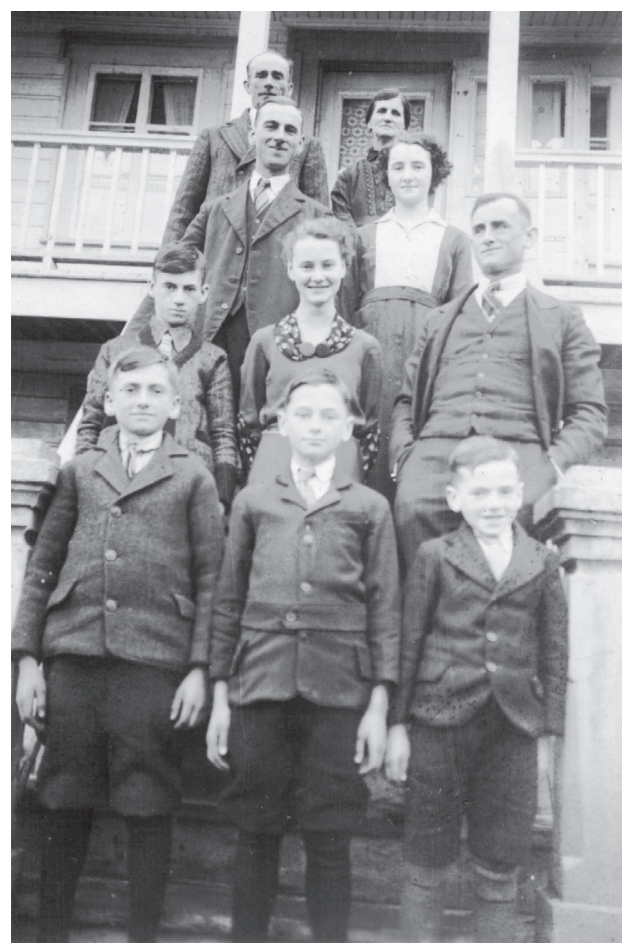

L'autorité s'exerce du haut vers le bas

À partir du haut, les parents : Arthur père et Demerise puis les enfants : Arthur fils, 27 ans (nommé Pierre dans St. Denis), Anne, 21 ans (Marie); Robert, 18 ans (Georges), Laure, 20 ans (Thérèse), Henri, 25 ans (Joseph) ; Georges, 13 ans (Thomas), Camille, 11 ans (Jean), Joseph, 7 ans (Henri).

de l'observation et la perspicacité de l'anthropologue puisque je ne saurais décrire la plupart de ces personnes mieux qu'il ne le fit, moi qui les ai pourtant intimement connues.

\section{Témoin de la vie rurale}

Miner photographiait aussi en vue d'échantillonner, de déceler une tendance, une norme ou de comparer. Il assistait aux travaux des champs aussi bien qu'aux tâches principalement dévolues aux femmes, comme celles du traitement et de la fabrication de la matière textile. Il fit de nombreux clichés de femmes en train de filer la laine ou de tisser, des fois chez les Garon, des fois chez les Raymond, des fois chez les Gagnon, des fois chez les Chouinard, des fois chez tous ces gens-là. Il allait s'en servir pour illustrer des passages de la monographie où il faisait ressortir la valeur économique de ces activités. Mais pourquoi autant de photos sur le même sujet? 


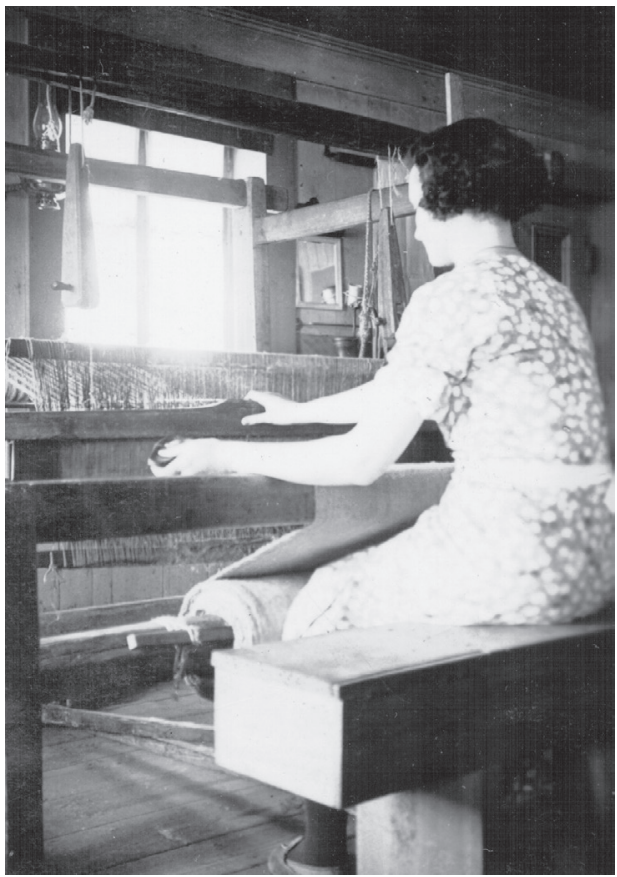

Ma tante Anne Gagnon au métier à tisser

Cette photographie n'apparaît pas dans St. Denis

Lorsqu'on compare la photo de tante Anne avec celle qu'Horace Miner a placée à la page 53 de St. Denis, on se rend compte que l'image utilisée dans le livre donne une vue bien plus précise du métier à tisser, que la tisseuse y présente son profil, que le décor de la pièce y est plus clair et, enfin, qu'il y a un rouet à l'avant-plan. L'auteur choisit donc l'image qui lui semble la plus typique, la plus éloquente, la plus représentative, celle qui illustre le mieux les lieux, les personnes, les décors et les outils normalement associés à l'activité, celle qui supporte le mieux son propos, celle qui projette le mieux sa vision de traits communs après comparaison.

Autre utilité de la photo, documenter toutes les étapes d'une tâche ou d'une opération en vue d'une description écrite dans la monographie. Que ce soit pour tendre une pêche à anguilles, fabriquer un four à pain, on trouve des exemples de séries photographiques où chacune des étapes du travail est photographiée. Pour le traitement manuel du lin en vue d'en faire une fibre textile exploitable, Miner se tourne vers mes grands-parents. Il tire plusieurs photos de l'activité même s'il n'en publie qu'une à la page 156A de St. Denis, "The écorçage of the flax ». Les clichés successifs lui fournissaient une vue de l'enchaînement des opérations et de la distribution des tâches selon le 
genre. Il pouvait ensuite décrire l'activité en détail et la rendre intelligible pour qui ne l'avait jamais observée.

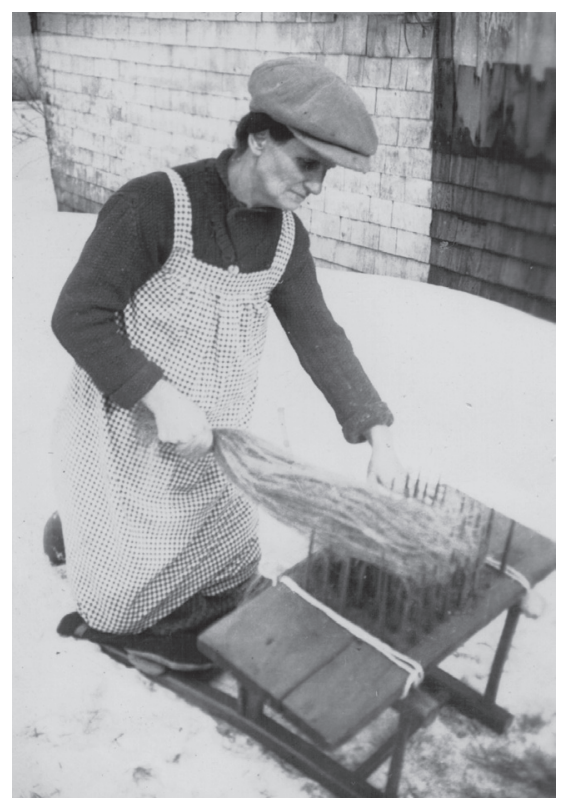

Ma grand-mère Demerise en train de peigner le lin À la page 156A de St. Denis, on la voit à l'étape de l'écorçage

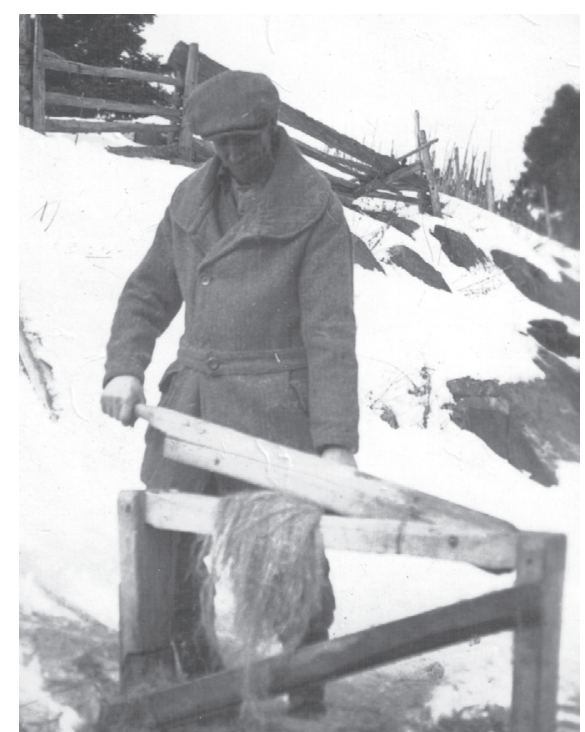

Mon grand-père Arthur en train de brayer le lin Cette tâche était remplie par les hommes du fait qu'elle exigeait une plus grande force physique 


\section{Des cartes de visites}

À part les photographies proprement documentaires comme les lieux, les monuments, les cérémonies religieuses ou les édifices, Horace Miner fit un grand nombre de portraits. Sans doute pour compenser l'embarras initial qu'il suscitait, on peut croire qu'il s'en servait pour apprivoiser les gens, pour faire accepter la présence de son indiscrète caméra. Il ne voulait certainement pas jouer les Edward S. Curtis puisqu'il n'inclut qu'un seul portrait dans St. Denis, «An old Canadien » en page 199A. En fait, si je prends l'exemple de la famille Gagnon, j'en viens à conclure qu'il se servait de ces images comme cartes de visite, voire de monnaie d'échange. Les albums assemblés par mes grands-parents contiennent des dizaines de photos qu'H. Miner leur avait données. Chaque fois qu'il photographiait un membre de la famille, soit en portrait soit en action, il en faisait finir un double qu'il remettait à l' « oncle Arthur ». Ainsi, les sujets avérés ou éventuels devaient accepter beaucoup plus facilement de se laisser photographier par la suite, qu'ils soient endimanchés ou non, qu'ils soient au travail ou au repos.

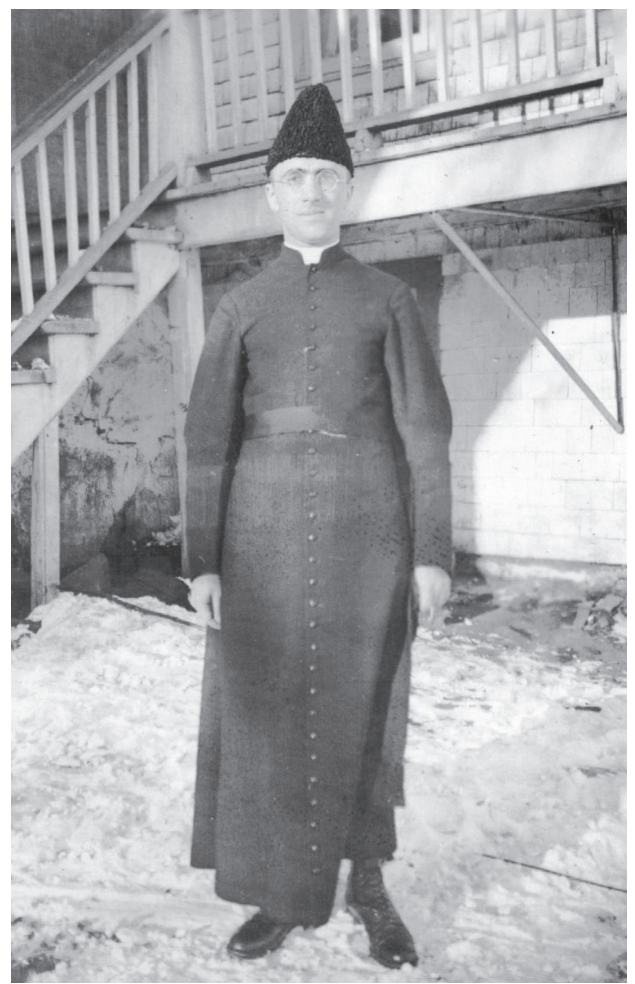

L'oncle François, aîné de la famille

prêtre et donc source de grande fierté pour ses parents 


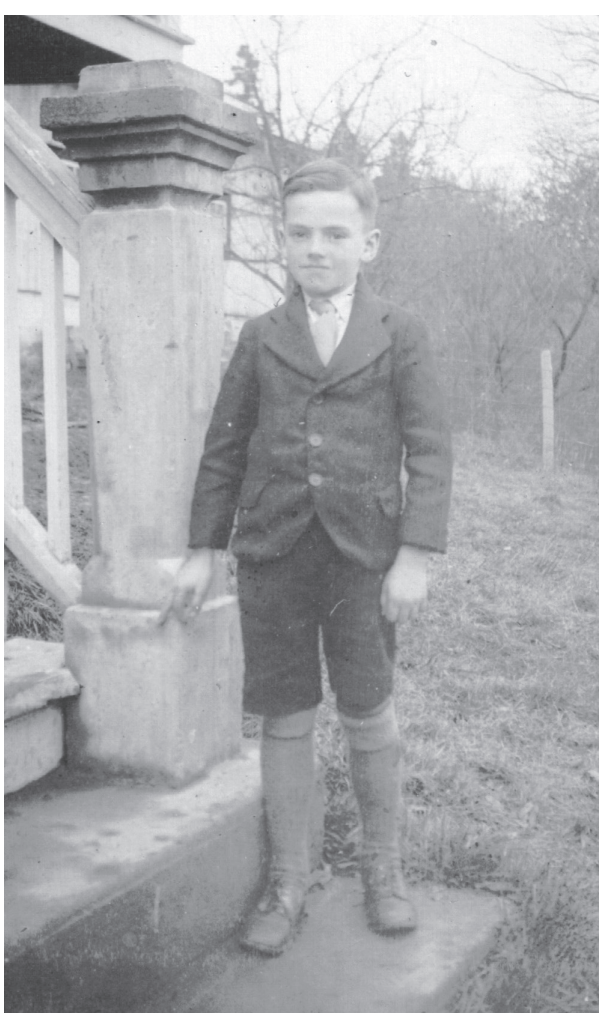

L'oncle Joseph, cadet de la famille

« d'un caractère heureux et gai ${ }^{10}$ », « il est chouchouté et gâté. ${ }^{11}$ »

Les portraits de l'oncle François et de l'oncle Joseph n'ont pas été faits pour publication. D'autant qu'en 1936-1937, l'oncle François n'habite plus la maison paternelle, mais le Collège de Sainte-Anne ; il n'a plus de rôle à jouer à Saint-Denis et, d'ailleurs, Miner n'en parle pas dans sa monographie sinon de manière allusive. Quant à l'oncle Joseph, les deux citations rapportées en légende, tirées du chapitre sur l'enfance, sont à peu près les seuls extraits où il en est question. Toutefois, Miner prenait la mesure du plaisir qu'il procurait à mes grands-parents en tirant ces portraits et en leur en remettant un exemplaire. Il se gagnait ainsi leurs faveurs et s'ouvrait encore un peu plus grande la porte de leur intimité. En 1936, les gens de Saint-Denis ne payaient pas souvent pour se faire tirer le portrait. Proposer de le faire gratuitement constituait sûrement une entrée en matière appréciée.

10. Saint-Denis, op. cit., p. 263.

11. Ibid., p. 266. 
En digne représentant de l'École de Chicago, l'anthropologue ne pouvait compléter une recherche aussi poussée sans inclure une histoire de vie. Celle-ci apparaîtra en appendice. Intitulée « Autobiography of an Habitant », elle reprend les faits marquants de la vie de mon grand-père, un homme que Miner avait jugé représentatif de sa communauté.

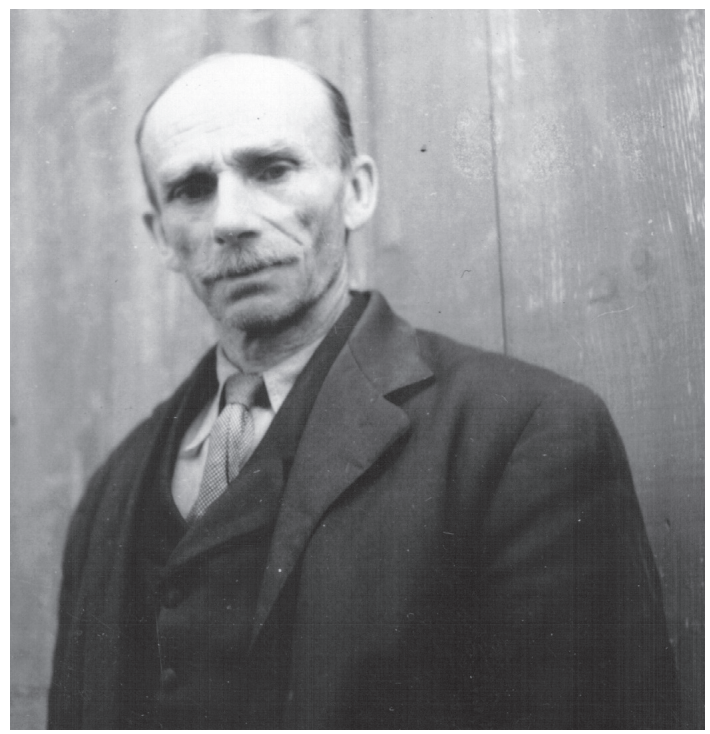

Mon grand-père Arthur

Horace Miner l'a aidé à raconter sa vie ${ }^{12}$

Sur la piste d'Horace Miner, Bernard Émond a dû lui aussi être frappé par l'abondance et la qualité de ces photographies. Après qu'Agnes Miner lui eut donné accès à des documents d'archives personnelles, il en exploite un bon nombre dans Le Temps et le lieu, dévoilant ainsi aux cinéphiles des images réservées jusque-là à une poignée de privilégiés. D'ailleurs, il faut remercier Agnes Miner et Bernard Émond d'avoir cédé ce trésor ethnographique au Centre d'archives de la Côte-du-Sud où des historiens, des ethnologues, des archivistes et autres chercheurs peuvent puiser une abondante matière à diverses publications. À titre d'exemple, l'historien Yves Hébert publiait en 2014 le livre d'images Kamouraska, dans le murmure du vent ${ }^{13}$ qui contient 16 photographies du fonds Horace Miner.

12. Ibid., p. 354 à 367.

13. Yves Hébert, Kamouraska, dans le murmure du vent, Québec, Éditions GID, « 100 ans noir sur blanc », 2014, 208 p. 


\section{L'anthropologue à l'épreuve du terrain}

En 1995, dans les actes de l'American Philosophical Society, dont Horace Miner était membre élu, l'auteur d'un hommage posthume à l'anthropologue évoque les suites de son séjour à Saint-Denis :

En 1936-37, après son mariage avec Agnes Murphy, le jeune couple passa près d'un an à Saint-Denis, un petit village situé à une cinquantaine [sic] de milles à l'est de Québec, sur la rive sud du Saint-Laurent, en vertu d'une bourse prédoctorale du Ssrc [Society Science Research Council]. Cette étude d'une communauté contemporaine au moyen de techniques anthropologiques constituait alors l'une des premières de ce type. Son travail de terrain produisit une thèse de doctorat, un livre - St. Denis : A French Canadian Parish (1939) - et une fille, Denise, née en $1938 .{ }^{14}$

Passons sur le cas de la fille, dont le prénom Denise relève d'une pure coïncidence selon une confidence de sa mère à Bernard Émond, pour plutôt examiner comment Miner lui-même fut marqué par ce premier terrain et quels furent ses rapports ultérieurs avec la famille Gagnon.

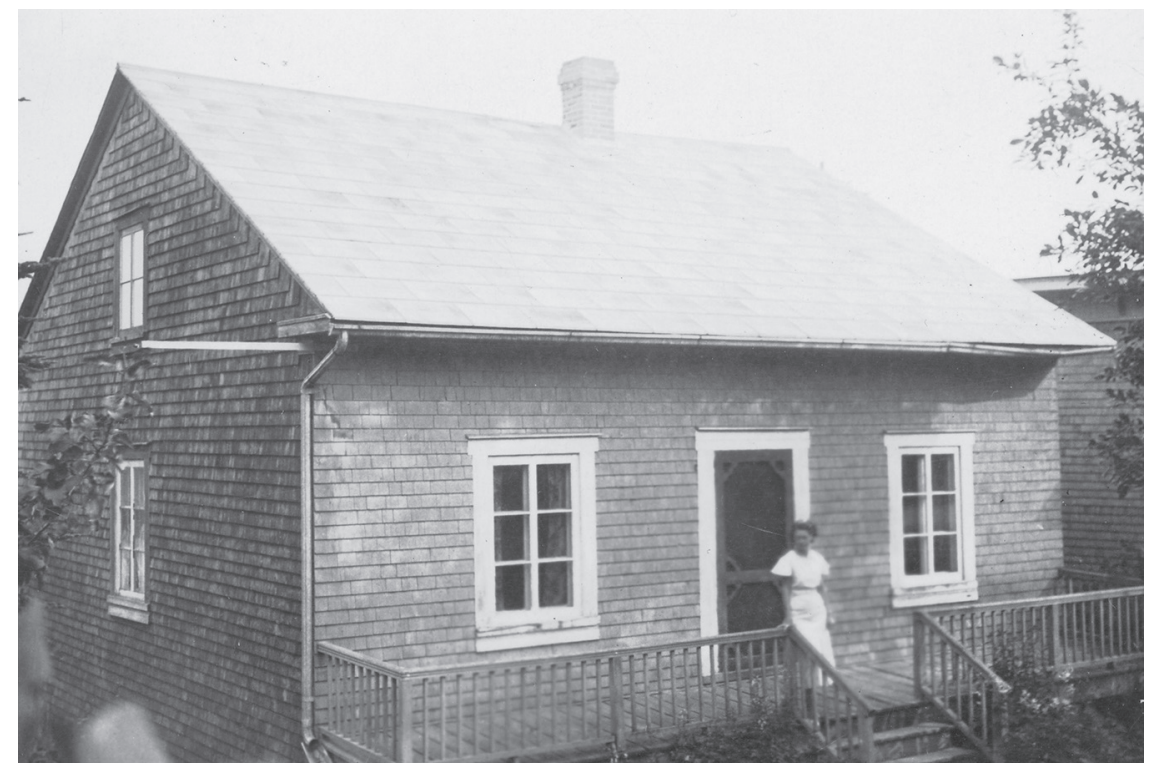

Agnes Murphy-Miner

devant la maison que le couple habita à Saint-Denis

14. James B. Griffin dans Proceedings of the American Philosophical Society, vol. 139, $\mathrm{n}^{\circ} 3$, 1995, p. 289 : "In 1936-37, following his marriage to Agnes Murphy, they spent about a year in St. Denis, a small town about fifty miles [sic] east of Quebec City on the east side of the St. Lawrence, on a predoctoral SSRC [Society Science Research Council] fellowship. His study of a modern community with anthropological techniques was one of the earliest such studies. His work there produced his doctoral thesis, a book, St. Denis : A French Canadian Parish (1939), and a daughter, Denise, in 1938. » [Traduction de l'auteur de l'article] 
Les premiers contacts que les deux jeunes Américains eurent avec la famille de mon grand-père furent assez saisissants s'il faut en croire Agnes Murphy-Miner, comme elle le rapporte dans l'article qui suit («Vivre avec le passé »). La maison qu'ils habitèrent tout au long de leur séjour de près d'un an appartenait à mon grand-père. La famille Gagnon fut donc une des toutes premières de celles avec qui ils eurent des contacts suivis dans le village.

Le film Le Temps et le lieu contient le précieux témoignage d'Agnes Miner sur les souvenirs qu'elle garde de son séjour à Saint-Denis et sur leurs relations avec la famille de mon grand-père. Inutile d'y revenir ici et de répéter ce qu'en dit le film.

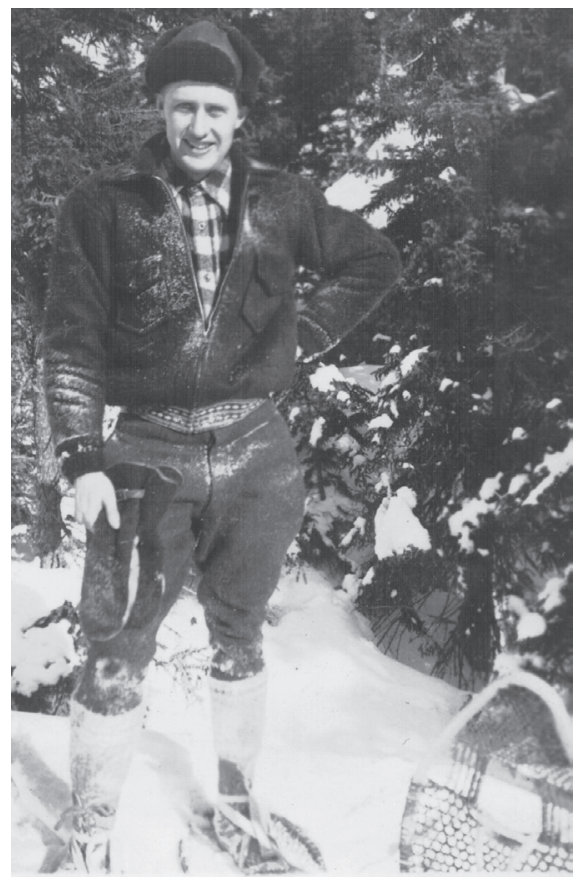

Horace Miner en habitant

avec culottes d'étoffe, bottes sauvages, raquettes et ceinture fléchée

Bien au-delà de la famille, il convient de percevoir l'attitude d'Horace Miner qui, en authentique pratiquant de l'anthropologie participative, cherche non pas à s'intégrer, mais au moins à s'imprégner de cette culture pour mieux l'analyser, la comprendre et la décrire. Agnostique, il fréquente assidûment l'église, il participe aux cérémonies, il pose des questions, reste constamment à l'affût du moindre événement digne d'intérêt. En d'autres termes, il semble prêt à tout pour s'initier aux coutumes de la paroisse. Par mimétisme, 
il n'hésite pas à se déguiser en homme des bois. Il ira même jusqu'à désigner sa compagne Agnes par le vocable " créature » : «Agnes is sure making a nice créature up here..$^{15}$ "

Bien que ce ne soit pas sa recherche à elle, Agnes, aussi anthropologue, aide son mari du mieux qu'elle le peut et joue le jeu avec lui. Elle l'accompagne dans toutes les activités où il convient de le faire.

Plus profondément que par les autres expériences de terrain qu'il fit, Miner resta marqué par son séjour à Saint-Denis. D'un point de vue professionnel, il y donna suite une douzaine d'années plus tard par un second terrain, de moins d'un mois cette fois, dont il publia les résultats dans The American Journal of Sociology ${ }^{16}$. Cette mise à jour constitue également la postface de l'édition de 1963 de St. Denis. Sur un plan personnel, Agnes Miner rappelle que le couple revint plusieurs fois à Saint-Denis au cours des années d'aprèsguerre $^{17}$. La fréquence de leurs visites diminua avec les années. Le début de cet article évoque celle de 1959. Puis Horace, Agnes et Denise revinrent en 1975. Cette fois, ils se heurtèrent à des portes closes. Ou presque. Mon grandpère Arthur était mort de même que ma grand-mère. Ils vinrent à la maison de mon père, mais celui-ci passait l'été à son chalet de Saint-Denis-sur-mer à un mille de la maison. Seule, ma sœur Irène qui se trouvait là par hasard put les rencontrer. Mais la conversation ne dura pas longtemps puisque ni l'une ni les autres ne partageaient plus une langue commune.

Comme dernier indice de la marque indélébile que lui laissa son séjour à Saint-Denis et les contacts avec mon grand-père, on ne saurait trouver mieux que le court article d'une page - cinq paragraphes -, empli de nostalgie, qu'Horace Miner écrivit pour annoncer sa retraite définitive dans LSA [Literature, Science and the Arts], un journal facultaire de l'Université du Michigan (Ann Arbor). Intitulé « Full Circle ${ }^{18} »$, il fait référence à mon grand-père et à Saint-Denis dans la majeure partie du troisième paragraphe :

Je reçois par la poste une demande de publier en traduction française la transcription du récit que me fit Mon Oncle Arthur, il y a longtemps, de sa jeunesse comme bûcheron à la frontière américaine [1'Appendice III intitulé Autobiographie d'un habitant]. Voilà quelqu'un qui veut ressusciter l'homme de la campagne et lui mettre en bouche l'idiome cultivé des Français. ${ }^{19}$

15. ACS, Fonds 241, Archives Horace Miner : lettre d'Agnes Miner à Bessie, 13 mars 1937 ; post-scriptum de la main d'Horace.

16. Horace Miner, «A New Epoch in Rural Quebec », American Journal of Sociology, vol. LVI, 1950, p. 1-10.

17. Voir l'article suivant.

18. Qu'on pourrait traduire par « Boucler la boucle».

19. Horace Miner, «Full Circle », LSA [Literature, Science and the Arts], Ann Arbor, University of Michigan, vol. 5, $\mathrm{n}^{\circ}$ 2, winter 1982, p. $8:$ « The mail brings a request to reprint, in French translation, my version of what «Mon Oncle Arthur » told me long ago of his early life as a woodsman on the Quebec frontier [L'appendice III intitulé Autobiography of an Habitant]. Now someone wants to resurrect 
Et en guise de conclusion, comme je ne saurais mieux dire qu'Horace Miner lui-même, je me permets de citer le dernier paragraphe du même article :

Un ami vient de m'appeler pour me raconter le mariage d'un couple qui, à la mode d'aujourd'hui, ont écrit les textes de la cérémonie. Ils y ont intégré un extrait d'une description que j'avais faite d'un mariage dans le village de Mon Oncle Arthur ! Bon, tout à coup ce que je croyais mort en même temps que lui vient de revivre. Et comme la tradition qui se travestit en nouveauté, il me tarde d'avancer vers le passé. ${ }^{20}$

\section{Vivre avec le passé ${ }^{21}$}

Agnes Miner

traduction par

BERNARD ÉMOND

- Pourquoi voulez-vous vivre dans notre village?

- Est-ce que vos parents sont encore vivants?

- Combien d'enfants avez-vous ? Combien de frères et sœurs ?

Ces questions et bien d'autres nous étaient posées dans le français des agriculteurs de la petite paroisse de Saint-Denis, au Québec. Nous rencontrions pour la première fois Monsieur Dieudonné Paradis ${ }^{22}$, un fermier prospère qui possédait la maison que nous voulions louer, et nous étions timides et maladroits. Nous n'étions mariés que depuis un mois et nous n'avions pas beaucoup confiance en nous au moment de commencer notre première recherche anthropologique sur le terrain. La famille étendue de Monsieur Paradis comptait vingt-huit personnes, de l'arrière-grand-mère au dernier bébé qui avait un mois, et tous nous regardaient avec curiosité, assis en demicercle autour de nous. On nous demanda si nous avions rencontré le curé.

- Oh oui. En fait, c'est lui qui nous a parlé de votre maison dans le village. Il pensait qu'elle pourrait nous convenir.

the rustic man by insinuating the cultured tongue of France into his untutored mouth. » [Traduction de l'auteur de l'article].

20. Ibid. : " A friend calls to tell me of the wedding of a couple who, in today's free style, composed their marriage lines themselves. Included was a passage I once wrote to describe a wedding in the village of Mon Oncle Arthur! Now, suddenly, all that I thought had died with him is resurrected, and, as tradition puts on the cloth of innovation, so can I look forward in the past. » [Traduction de l'auteur de l'article]

21. Madame Miner a écrit ce texte, qui n'a jamais été publié, à son retour de Saint-Denis en 1937. Le titre original était : Living with Yesterday. Les noms des personnes mentionnées dans le texte sont pour la plupart fictifs.

22. Arthur Gagnon, de son vrai nom. 
Puis on nous demanda si nous allions à la messe régulièrement. Les gens parurent satisfaits quand mon mari dit que nous aimions particulièrement la maison de M. Paradis parce qu'elle était située en face de l'église et de la salle paroissiale. Il y eut un autre long silence, ponctué par le craquement des chaises berçantes sur le plancher de linoléum. Comme ce n'était pas une visite « officielle », nous étions réunis dans la grande cuisine d'hiver. Nous aurions été encore plus intimidés par le salon, avec ses décorations funéraires de couronnes de fleurs séchées, ses médaillons de cheveux tressés, la grande croix de tempérance et les portraits sévères des ancêtres disparus. Finalement, Papa Paradis (comme nous allions bientôt l'appeler) nous dit que nous pouvions avoir sa petite maison. Il nous considéra avec circonspection quand nous lui demandâmes s'il pouvait nous bâtir un foyer avec une cheminée.

- Comment? dit-il.

- Une cheminée.

- Qu'est-ce que c'est?

Aussitôt que mon mari se mit à expliquer, il y eut un éclat de rire général et quelqu'un dit :

- Ah! Vous voulez dire une chûnée !

Tout le monde trouvait cela très drôle, non seulement à cause de notre façon étrange de parler français, mais surtout parce qu'il n'y avait rien de plus ancien et démodé qu'un foyer. Comment pouvait-on vouloir un foyer alors qu'on avait d'excellents poêles à bois, émaillés et nickelés, qui donnaient efficacement une bonne chaleur. Nos amis ne trouvaient pas que notre idée d'un foyer, qu'on finit par construire à nos frais, était bonne. Pour eux, c'était une excentricité et ils nous le dirent souvent. Les femmes, par contre, s'intéressaient à notre poêle et, même maintenant, je me souviens de ma fierté quand elles me dirent :

- Votre poêle est bien propre.

Cela signifiait qu'elles me considéraient comme une bonne ménagère. À chaque fois que Madame Barbeau ou Mademoiselle Dionne, la ménagère du curé, venaient en visite, le poêle était toujours le premier sujet de conversation. Nous discutions de la quantité de bois que je brûlais, du nombre de pains qu'on pouvait cuire au four et si le poêle gardait bien sa braise pendant la nuit. J'en vins à aimer ce poêle, qui était le centre et le point focal de la maison. Il nous gardait au chaud et nous faisait des amis. Je crois que nous aurions besoin de ce genre de symbole dans nos maisons américaines.

Les familles étaient nombreuses, avec en moyenne dix enfants. Les Paradis en avaient dix-sept, même si Madame Paradis était une femme menue qui ne pesait pas cent livres. Comme la plupart des femmes du village, elle n'avait pas recours à un médecin pour ses accouchements. Quand quelqu'un se cassait un bras ou une jambe, Papa Paradis envoyait chercher le raman- 


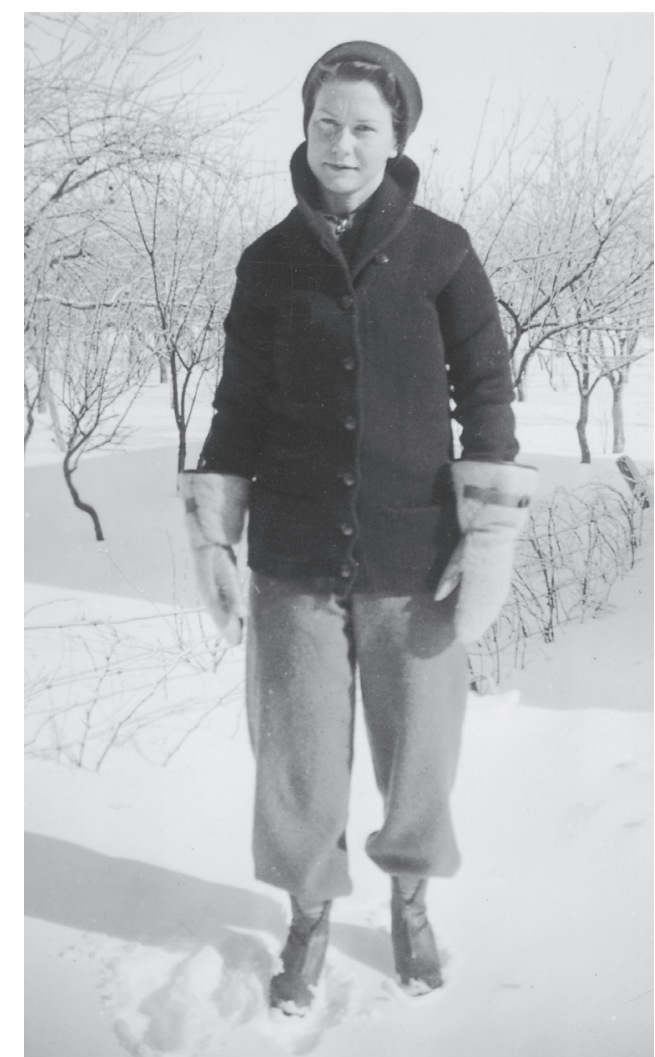

Agnes Murphy-Miner, hiver 1937 à Saint-Denis

Photo : Archives de la Côte-du-Sud

cheur. Très souvent, le ramancheur était aussi vétérinaire et il était compétent dans ses deux métiers. Il pouvait recommander toutes sortes d'herbes et de remèdes pour différentes maladies. Les gens pouvaient aussi compter sur les demoiselles Lévesque pour donner un coup de main pendant une maladie. Il fallait être en danger de mort pour qu'on décide de faire venir le médecin du village voisin. Il arrivait que le glas sonne déjà au clocher de l'église avant que le médecin ne soit arrivé. Et quand le glas sonnait, tous s'arrêtaient et comptaient le nombre de coups. Un coup sur chacune des trois cloches pour un enfant, deux pour une femme et trois pour un homme, le tout répété trois fois. Les gens disaient une prière en mentionnant le nom du défunt ; on savait généralement qui il était, dans cette communauté tricotée serrée. Les funérailles étaient un événement pour toute la paroisse, comme d'ailleurs les mariages. Avant la cérémonie, on se demandait s'il s'agirait de funérailles de première, deuxième ou troisième classe. Les paroissiens aimaient le 
décorum d'un enterrement de première classe, avec le corbillard conduit par un directeur de funérailles portant haut de forme et redingote, et suivi par une procession solennelle de gens vêtus de noir, prêtres et famille du défunt.

Nous trouvions l'hiver interminable et une neige lourde et profonde couvrait le sol d'octobre à mars. Mais les mois d'hiver étaient les plus agréables, alors que les agriculteurs n'avaient pas de travaux pressants et que les femmes n'étaient plus occupées à leur jardin et leurs conserves. C'était enfin le temps des veillées et des mariages. Souvent, après la messe du dimanche, nous montions dans un traîneau à cheval gaiement décoré, nous nous blottissions sous la peau d'ours et nous traversions les champs enneigés pour nous rendre à une ferme. Aussitôt que les gens entendaient les grelots du traîneau, ils sortaient de la maison pour nous accueillir. Nous étions maintenant habitués à des familles de vingt ou trente personnes. Après la promenade en traîneau, c'était un délicieux repas composé de poulet, de conserves de légumes et de pain maison. Après, les hommes bourraient leur pipe de fort tabac canadien et fumaient, pendant que les femmes desservaient et papotaient entre elles. Parfois, on couchait les jeunes enfants pour une sieste, mais il n'y avait pas de règle établie : les enfants pleuraient rarement et ne faisaient pas de caprices.

Comme les paroissiens de Saint-Denis faisaient partie d'une société de tempérance, fondée en 1870 , on ne buvait pas. S'il y avait un musicien dans la compagnie, on se mettait à chanter au son du violon ou de l'accordéon. Autrement, on jouait au quatre-sept ou on discutait de politique, de récoltes ou des fermes qui étaient à vendre. Souvent, le curé était présent. Bien qu'il fût l'objet d'un grand respect, les fermiers n'hésitaient pas à différer d'opinion avec lui en dehors des questions religieuses. Comme ils savaient que nous serions intéressés, nos amis nous montraient des cahiers calligraphiés contenant leur généalogie. Nous pouvions y lire le nom des pionniers venus de Normandie au XVII ${ }^{\mathrm{e}}$ siècle pour se tailler une vie nouvelle sur les rives vierges du Saint-Laurent. Nous sentions bien la fierté que Monsieur Paradis, par exemple, éprouvait à l'égard de l'histoire de sa famille. Il aimait raconter des anecdotes à propos d'un grand-père, d'un oncle ou d'un frère, à propos des anciennes batailles avec les Anglais ; ou comment un de ses parents défrichait courageusement une terre de colonisation en Abitibi.

Il y avait une fierté dans le dur labeur qui produisait les champs de blé et de lin, les moutons gras, le bétail, les cochons, les jardins bien nettoyés des femmes, les hautes cordes de bois bûché et coupé par le fermier et ses fils. Nous comprenions que ce qui au début nous avait semblé une vie de travail sans fin et sans joie était une vie pleine de récompenses et de satisfactions pour les gens de Saint-Denis. Nous nous mîmes à respecter et à admirer un mode de vie maintenant rare dans notre propre société. C'était comme voyager 
dans le passé, le genre de passé qui avait dû exister auparavant aux États-Unis. Chacun ici avait des valeurs inébranlables, sans les incertitudes qui caractérisent notre culture urbaine américaine. C'est peut-être ce qui expliquait l'absence complète de divorces, de dettes, de vols et de meurtres. Nos amis n'avaient pas besoin de magasins et de marchandises. Ils produisaient toute leur nourriture, portaient des vêtements faits avec le lin de leurs champs et la laine de leurs moutons. Pendant les longs hivers, les femmes s'assoyaient à leurs rouets et à leurs métiers pour fabriquer des tapis, des couvertures, des couvre-lits. Pour la plus jeune fille comme pour la plus vieille femme, il y avait toujours des bas et des tuques à tricoter, des catalognes à fabriquer, un bébé à dorloter.

Noël fut merveilleux. Nous allâmes dans la forêt pour chercher un sapin que nous décorâmes avec des chaînes de papier de couleur, de maïs soufflé ou d'atocas, et des noix enveloppés dans du papier de plomb. Nous taquinions nos amis en leur disant que nous serions les seuls à recevoir des cadeaux du Père Noël puisque nous avions la seule cheminée du village ! La veille de Noël, plusieurs personnes vinrent voir notre sapin et nous souhaiter joyeux Noël. Il y eut un moment gênant quand deux familles d'opinions politiques opposées arrivèrent en même temps. Elles refusaient de se parler depuis des années, mais l'esprit des fêtes calma les esprits.

Quand vint le temps de quitter Saint-Denis, nous vîmes combien les villageois avaient été sensibles au fait que nous les avions aimés. Nous fûmes couverts de cadeaux. Il y avait des couvertures tissées à la main, faites avec la laine de moutons que nous avions connus; des linges à vaisselle faits de lin qui venait de champs semés et récoltés devant nous; des pommes qui venaient de vergers familiers; des mitaines tricotées par trois vieilles filles qui faisaient notre lavage avec le même soin qu'elles mettaient à entretenir les nappes de l'autel. Presque chaque été, nous retournons à Saint-Denis et chaque visite apaise notre esprit. Le vieux curé est parti, mais un nouveau vient combler la place laissée libre. Il y a bien sûr des changements faits au nom du progrès, comme l'eau courante et les tracteurs, mais les valeurs et l'amitié restent les mêmes. 